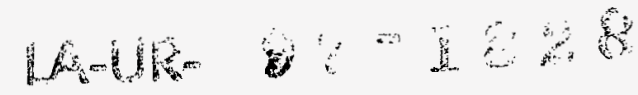 Conf-97/15
}

\section{An Object-Based Methodology For Knowledge Representation In SGML}

\author{
Robert L. Kelsey \\ Los Alamos National Laboratory \\ New Mexico State University \\ rob@lanl.gov \\ Robert B. Webster \\ Los Alamos National Laboratory \\ robw@lanl.gov \\ Roger T. Hartley \\ New Mexico State University \\ rth@cs.nmsu.edu

\section{4} \\ RECEIVED \\ AUG 131997 \\ OSTI

\begin{abstract}
An object-based methodology for knowledge representation and its Standard Generalized Markup Language (SGML) implementation is presented. The methodology includes class, perspective, domain, and event constructs for representing knowledge within an object paradigm. The perspective construct allows for representation of knowledge from multiple and varying viewpoints. The event construct allows actual use of knowledge to be represented. The SGML implementation of the methodology facilitates usability, structured, yet flexible knowledge design, and sharing and re-use of knowledge class libraries.
\end{abstract}

\section{Introduction}

An object-based methodology for knowledge representation uses objects of classes as its fundamental representational building block. The overall concept of using objects as a building block is not unlike what is done in object-oriented analysis (OOA) and design (OOD) [3] [2]. Each of these decomposes knowledge into manageable units called objects. It is natural to decompose knowledge into objects and objects are modular enough to allow for changes and extensions within the representation. 
This methodology is a design methodology (not a representation system) for designing domains of knowledge. Knowledge representation systems often skip the step of designing the knowledge that the system represents. As a result, knowledge representation systerns are difficult to use. It may be possible that knowledge designed using this methodology can later be put into existing knowledge representation systems.

This methodology includes the use of perspectives and events. Perspectives allow for the representation of knowledge from multiple viewpoints. This can increase the detail of the knowledge and put the knowledge in additional context. Events allow for the representation of actual use of the knowledge along with the knowledge itself.

The use of Standard Generalized Markup Language (SGML) to implement the methodology adds structure, but maintains flexibility. Also, SGML facilitates the sharing and re-use of knowledge through it portability and constructs. A less obvious, but important aspect of the SGML implementation is its added usability. A growing familiarity of markup languages helps make the SGML implementation of the methodology more usable. The following sections describe the methodology (briefly) and the SGML implementation. More detail about the methodology can be found in [6].

\section{Methodology Background}

\subsection{Introduction}

Knowledge is represented in the methodology using three different constructs, the class, the domain, and the event. A class is a template or prototype that describes an object or collection of objects. Once a class is defined it is an available resource for creating a domain of knowledge. A domain is a specific focus of knowledge. Classes are instantiated for use in a domain. Instantiation causes specific instances or objects of those classes to become available for use. This is why the domain can be focused, because only the classes of interest or pertinence need be instantiated. An event is a construct for representing examples of actual use of the knowledge in a domain. 


\section{DISCLAIMER}

This report was prepared as an account of work sponsored by an agency of the United States Government. Neither the United States Government nor any agency thereof, nor any of their employees, make any warranty, express or implied, or assumes any legal liability or responsibility for the accuracy, completeness, or usefulness of any information, apparatus, product, or process disclosed, or represents that its use would not infringe privately owned rights. Reference herein to any specific commercial product, process, or service by trade name, trademark, manufacturer, or otherwise does not necessarily constitute or imply its endorsement, recommendation, or favoring by the United States Government or any agency thereof. The views and opinions of authors expressed herein do not necessarily state or reflect those of the United States Government or any agency thereof. 


\subsection{Class}

There are two types of classes possible in the methodology, agent and non-agent. Although the types are not formally designated in notation, the distinction is useful for determining interactions of and between objects of classes. Others (particularly Schank [10]) have used the same distinction in representation, calling agent objects actors. Agent objects of a class act on or initiate actions on other objects of both agent and non-agent classes. Non-agent objects of a class are essentially inanimate objects.

A class consists of three parts, attributes, methods, and perspectives. An attribute is a property or characteristic that helps describe an object of a class. An attribute has a name or identifier and a value. It may also have a list of potential or limiting values. The value of an attribute is assigned at the time an object is instantiated from a class and can be changed by methods of the object.

A method determines the behavior of an object of a class. A method is an allowed operation on an object. Objects interact with one another through methods and limiting the methods available can constrain interaction between objects. A method has a name or identifier, a list of arguments (if any), and the operation content. The operation content is how a method goes about doing what it does. This may be represented in a variety of ways including rules, pseudocode, or even a program. A method is not available for use until an object is instantiated from a class.

A perspective defines how or in what way an object of a class may be viewed. Some attributes may be important to one perspective while not important to another perspective. Thus, a particular perspective is defined by grouping the attributes and methods in a class that are pertinent to the perspective. Similar to a method, a perspective or an object is defined in that object's class and limits or constrains the available views. Each attribute and method in a class has a list of perspectives with which it is associated.

The name or identifier and the definition of a perspective go in the class that possesses that perspective or view. There is also a list of classes that are a part of this class's perspective. A perspective can be considered to be how one object of a class views the other objects in a domain. 


\subsection{Domain}

A domain is where objects of classes are instantiated for use. At instantiation, objects are created and named and values within the parts of an object are assigned. Only the knowledge necessary for a particular application need be defined in a domain. A domain has a name and a list of objects contained within the domain. An object (within the domain definition) has a name or identifier, the name of the class from which it was instantiated, and if it is an agent object, the perspective with which it is viewing the rest of the domain.

\subsection{Event}

A domain of objects can contain or represent knowledge and maybe even how the knowledge is used, but it cannot necessarily represent actual examples of use. An event is specifically used for representing an example use of knowledge. An event has a name or identifier and consists of four parts, a before state, a method, an after state, and an agent. A state is a snapshot of an object containing the values of an object's attributes at a particular moment in time. A method is the name of the method causing the change in state from before to after. An agent is the name of the agent object instigating the method to change the state.

Multiple objects may be involved in an event, but only those involved are represented in the event construct. Additionally, only the object attributes that change values from before to after state are listed within the states. This is in keeping with the spirit of only representing what is necessary.

\section{SGML Background}

\subsection{Introduction}

The Standard Generalized Markup Language (SGML) is an ISO standard for the description of documents [5]. This standard describes technology for facilitating text interchange in documents [11], but the 
technology has many potential uses. SGML is used to describe the structure and organization of a document, or more specifically, the structure and organization of the content of a document. As a result, the format of the content of a document and the content of a document are two separate things. In short, SGML enables the design and implementation of document structure and the associated notation for marking the content of a document.

Although SGML was originally designed for the publishing of both paper and electronic documents, it has been exploited for use in other areas including enterprise management, engineering, product life cycle, and multi-media. Any application that must process and manage information may benefit from SGML. SGML can help enable applications that use indexing and retrieval of information, cross reference of information, modification and revision of information, and multiple display formats.

\subsection{SGML Components}

There are three components or parts to an SGML document [1]. The first component is the SGML declaration which determines the formal syntax and any optional features to be used within documents. These are the rules for designing a document type definition (DTD) and for validating the conformance of a DTD and an associated document.

The second component is the document type definition (DTD) which is a rule set for a class or group of documents. The rules in a DTD describe the notation for marking the content pieces of a document and how those pieces relate to one another. Some example classes of documents are project plans, memos, and technical reports. Each of these classes of documents has its own style and format.

The third component is an instance of a document which is an actual document containing content and markup. For example, a memo document may contain content like who the memo is to, who the memo is from, and a message. Markup for this document will label or tag those corresponding parts, the to, the from, and the message. 


\subsection{SGML DTD}

There are four constructs used in creating a DTD, the element, the attribute, the entity, and the comment [7]. Note that the attribute construct should not be confused with the attributes of a class discussed previously. The DTD is a grammar that defines the parts to a document, their structure, and their notation in terms of the four constructs mentioned previously.

An element construct can be thought of as a container [7]. Any information that is to be contained must have an element defined to hold the information. For example, the message portion of a memo document will have a message element. There may also be to and from elements. These elements hold and contain the content of the message portion, to portion, and from portion (respectively) of the memo document.

An attribute construct is attached to an element construct and is used to provide further detail about the element. For example, the message element may have an attribute called security type. The security type attribute designates whether the message is unclassified, sensitive, or classified. An attribute construct may be thought of as a modifier of the element construct.

An entity construct is used to contain portions of document content that are repeated throughout the document. By defining an entity, content is defined once, but can be used many times. In this way, an entity is like a macro or an include file. If the content changes, it must only be changed in one place. A comment construct is used to express comments or notes that are not part of the document content and not meant for the end user to see.

\section{Implementing the Methodology in SGML}

Implementing the object-based methodology in SGML involves creating a DTD that maps the methodology constructs and their parts into SGML constructs. The following sections describe the SGML constructs for the three methodology constructs and their parts. In the following sections note that when the word attribute is used it refers to the attribute part of the class construct in the methodology. An SGML attribute for an SGML element will be referred to as SGML attribute construct. Names of the created 
SGML constructs (and example values) are shown in boldfaced sans serif type. Names and values in the examples in figures are shown in proper SGML notation. The (uncommented) DTD created for the object-based methodology is in Appendix A.

\subsection{The Class Element}

The class construct becomes an element called class. The three parts to a class (attributes, methods, and perspectives) each become elements as well. Contained within the class element are the elements called attribute, method, and perspective. The attribute element may contain an element called potvals. The potvals element contains a list of potential values that an attribute may possess. The class and attribute elements each have an SGML attribute construct attached to them called name. In both, the name holds the name of the class and the name of the attribute, respectively.

The method element may contain the elements called args and content. The args element contains a list of arguments (if any) to the method. (Any elements that contain lists may use an additional markup called $i f$ to designate an individual list item.) The content element contains the operation content of the method. The method element also has an SGML attribute construct attached to it called name which holds the name of the method. The method and attribute elements each have an additional SGML attribute construct attached to them called perspect. In both, perspect holds a list of perspectives with which the method and attribute (respectively) are associated and/or grouped.

The perspective element may contain an element called def. The def element contains the definition of the perspective. There are two SGML attribute constructs attached to the perspective element called name and classes. Name holds the name of the perspective and classes holds a list of classes that are observed within this perspective or view.

\subsection{The Domain Element}

The domain construct becomes an element called domain. The domain element may contain an element called instance. Instance refers to an object being instantiated from a class. The domain element has an 
SGML attribute construct attached to it called name which holds the name of the domain. The instance element has three SGML attribute constructs attached to it called classname, name, and perspect. Classname holds the name of the class from which the instance or object is being instantiated. Name holds the name of the instance or object. Perspect holds the name of the perspective this instance or object uses to view the rest of the domain. Within the domain element is an element called attval. The attval element contains the actual value of an attribute. The attval element has an SGML attribute construct attached to it called name. Name holds the name of a particular attribute in the class.

\subsection{The Event Element}

The event construct becomes an element called event. The event element may contain four elements called befstate, meth, aftstate, and agent. The befstate element contains information about the before state. The meth element contains the name of the method causing the change in state. The aftstate element contains information about the after state. The agent element contains the name of the agent object that instigated the method to change the state. The event element has an SGML attribute construct attached to it called name which holds the name of the event.

\section{An AI Example}

To illustrate how the SGML implementation is used and what it looks like, a classic problem from artificial intelligence (AI) will be presented. The blocks world is one of the classic problems in AI [8] [9] [12] and comes from a collection of problems known as microworlds [9]. Microworld problems have limited knowledge domains and appear to need intelligence to solve.

The blocks world contains a table, blocks, and a robot arm. The robot arm manipulates the blocks into different arrangements on the table, such as stacks of blocks. The blocks world is a good example to illustrate this methodology and the SGML implementation because it is a narrow domain of knowledge, but contains enough to exercise most of the methodology's constructs. 
Note that the figures illustrating each of the constructs use the notation and markup as defined by the SGML DTD created for the methodology. If this research was about document construction and not knowledge representation, then the examples shown in the figures would be known as document instances. The markup language has an opening and closing tag for each element (methodology construct). An opening tag is delimited by the $<$ and $>$ symbols while a closing tag is delimited by the $<$ /and $>$ symbols. The name of the element (methodology construct) appears between the tag delimiters. If the element has an SGML attribute construct attached to it, then the SGML attribute appears (in quotes) in the opening tag of the element.

\subsection{Class Definitions}

The blocks world has three classes of objects which are table, block, and robot arm. The class for table is the simplest because it contains no attributes or methods. In this domain of knowledge, the table is merely a physical object where the blocks may be located. Defining more than this in the class table is extraneous and irrelevant. The class robot-arm has one attribute named holding. The holding attribute contains the knowledge of whether the robot arm is holding a block and if so, which block. The holding attribute may have potential values of NULL (meaning not holding a block) or a block's identification (ID).

The class robot-arm is an agent class, so it contains a perspective named stacking whose view of the domain includes the classes of table and block. The definition of the perspective is the typical view of the blocks world. Figure 1 shows the class definitions for table and robot-arm.

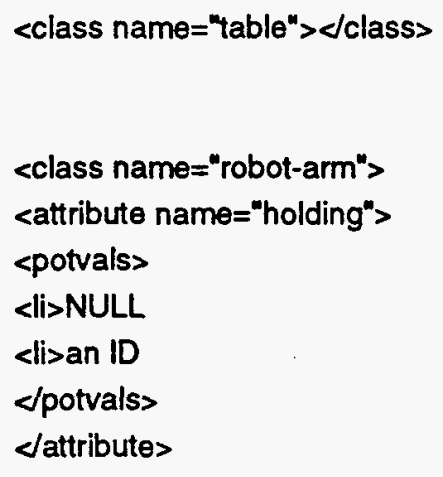

Figure 1: Class definition for classes table and robot-arm. 
class robot-arm was tried. The result was that it did not seem to matter. The methods can be put in either class, but it is important to select and use one convention consistently. One possible advantage to having these methods in the class block is that they act as a constraint. These are the only operations allowed on a block. The content of the methods is also shown in Figure 2.

\subsection{Domain and Event Definitions}

An example domain for the blocks world contains a table object named table1, a robot-arm object named robot-arm1, and four block objects named blockA, blockB, blockC, and blockD. The name of the domain is blocks-world1. The object robot-arm1 is instantiated with the value NULL for its attribute holding. Robot-arm1 is an agent object and views the rest of the domain from the stacking perspective (perspect). The four block objects are each instantiated with the values of their respective names for the attribute ID and the values table1 and NULL for the attributes location and covered, respectively. Figure 3 shows the definition of the domain blocks-world1.

An example event named event1 illustrates blockA being picked up by robot-arm1. The befstate shows blockA's location to be on table1 and robot-arm1 is not holding a block. The meth pickup of blockA is executed. The aftstate shows blockA's location to be robot-arm1 and robot-arm1 is holding blockA. The agent instigating the method is robot-arm1. Figure 3 shows the definition of the event event1.

\subsection{Another Perspective}

The SGML implementation of the methodology allows for easy extension to the existing knowledge. Suppose that in addition to the stacking perspective of the blocks world there is a perspective to teach a child to spell. In this new perspective the blocks may be alphabet blocks and words are spelled by placing the blocks next to each other. This new perspective is named spelling and will add attributes and methods to the existing class block. Also, the labels of which attributes belong to which perspectives will change.

In the class block there are two new attributes called rightside and leftside which may have potential values of NULL (meaning no block present to the particular side) or a block's identification (ID of block on 


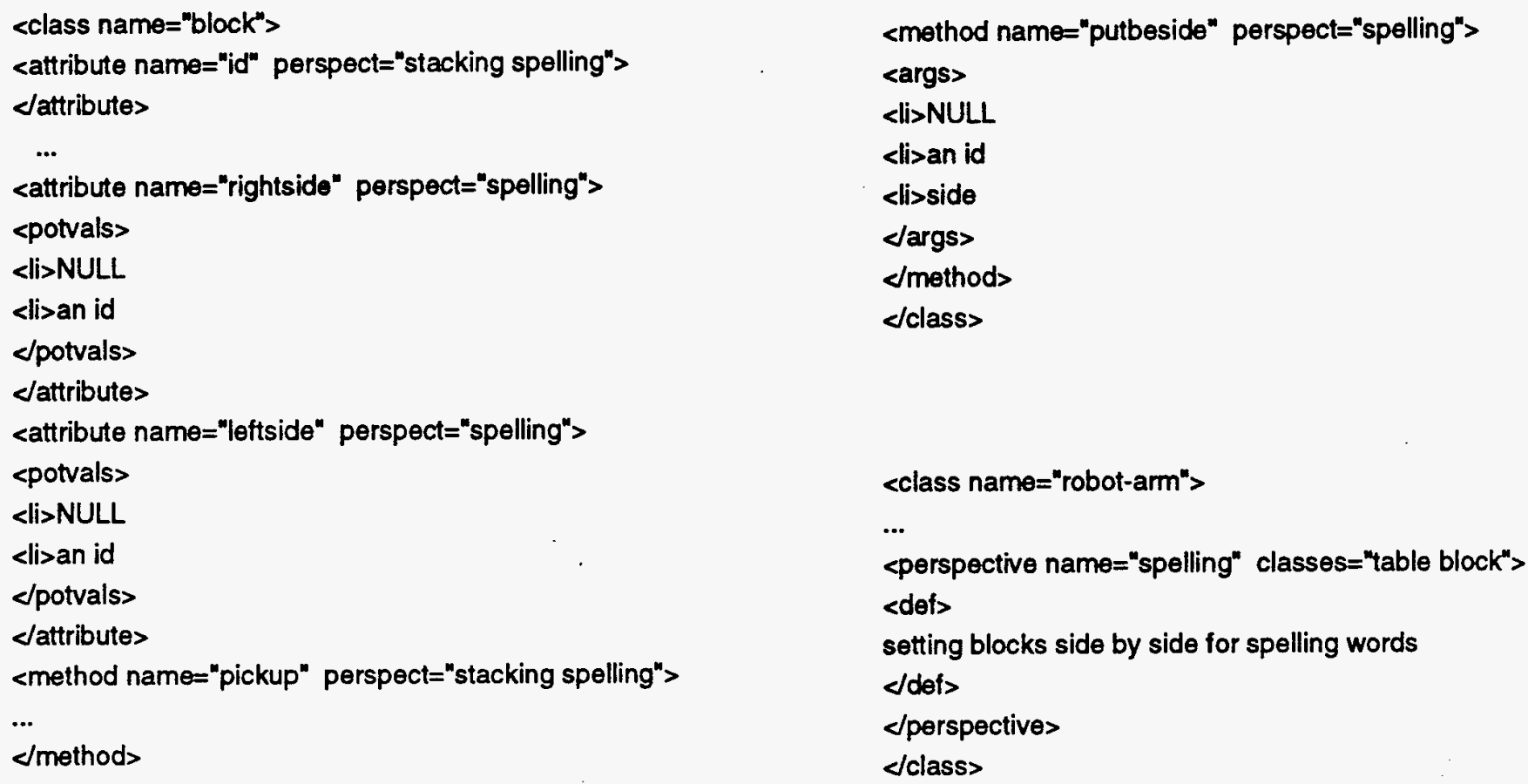

Figure 4: Definitions of class block and robot-arm with the added perspective spelling.

\subsection{Potential Reasoning}

Lines of reasoning are possible using perspectives and events. Different perspectives on the same object and the same perspective on different objects may suggest useful comparisons and contrasts. Gentner [4] describes comparisons of objects and domains based on literal similarity, analogy, and abstraction which are derived from the mappings of objects, object attributes, and relations between objects. Consider the example of the blocks world with the two different perspectives, stacking and spelling. These are two different views of the same sets of objects. It is fairly simple to observe that a stack of blocks laid down will look like a line of blocks side by side, but can a reasoning mechanism make this observation? Perspectives may help facilitate this type of reasoning.

Events may be useful for another potential line of reasoning. Consider the event event1 from Figure 3. Suppose the method (meth) was left blank. Given the before state (befstate), after state (aftstate), and class definitions (with method contents) it may be possible to infer the method (meth) executed to change the state. Actually, given any two of the three (befstate, meth, aftstate), the third may be inferred with 
the help of the class definitions and their method contents. It may be possible to make other inferences by including the agent with the other event elements.

Although not discussed in this paper, the methodology and its SGML implementation have the ability to create classes using the concepts of inheritance and aggregation/disaggregation. More detail about these concepts is discussed in [6]. Using inheritance and aggregation/disaggregation relationships can allow for additional lines of reasoning.

\section{Other Features}

There are some other features to the methodology that are made available by the SGML implementation. One of these features is the ability to share and re-use knowledge. Part of this is facilitated by the notion of containing knowledge in classes and part of it is facilitated by SGML. Class libraries of common knowledge may be created and distributed in an SGML form. A class can be defined within an entity construct in a DTD. This will allow the class to be included and used in other knowledge domains by referencing the defined entity. That fact that SGML is very portable will also contribute to the sharing of knowledge.

Another feature is the potential ease of use of creating representations using SGML. The increasing popularity of the World Wide Web (WWW) has increased the use and understanding of markup and tagging languages. Using SGML to implement the methodology exploits this growing familiarity with markup languages. In fact, in order to make the creation of knowledge (in SGML) easier and quicker for testing the methodology, an Emacs mode for GNU Emacs was developed. The mode has control-key sequences defined for all the methodology constructs and their associated tags. Additionally, a knowledge domain and the DTD for the methodology can be checked (within Emacs) against a validating SGML parser. In the future, code will be added to the Emacs mode to reason across some of the knowledge. 


\section{Conclusions}

The object-based methodology and its SGML implementation complement each other. Together they provide a structured, yet flexible means of representing knowledge. The available constructs allow knowledge to be limited and focused to a specific problem or application area. Only the pertinent knowledge need be represented. Should the focus later change, the existing knowledge can be easily changed and/or extended.

The perspective and event constructs add detail to the knowledge being represented. Perspectives allow for the representation of multiple and varying viewpoints. Events allow for the representation of actual knowledge use. Both these constructs may facilitate new lines of reasoning over the knowledge domain.

Additionally, the SGML implementation helps make the knowledge more usable and portable. SGML documents can be interchanged independent of hardware and software and there is no proprietary format [11]. Also, by using the SGML entity construct, class libraries of common knowledge can be created, shared, and re-used.

More research is yet to be done on exercising the methodology and its SGML implementation on more complex knowledge domains. The limits of the methodology in what types of knowledge it may represent must be identified. Also, it should be compared to other representation schemes and systems. More research must also be done to further understand and implement the potential reasoning mechanisms in the methodology including reasoning with perspectives and events.

\section{References}

[1] Liroa Alschuler. ABCD...SGML A User's Guide To Structured Information. International Thomson Computer Press, Boston, MA, 1995.

[2] Grady Booch. Object-Oriented Analysis and Design with Applications. Addison-Wesley Publishing Company, Menlo Park, CA, second edition, 1994. 
[3] Peter Coad and Edward Yourdon. Object-Oriented Design. Yourdon Press, Englewood Cliffs, NJ, 1991.

[4] Dedre Gentner. Readings in Cognitive Science A Perspective from Psychology and Artificial Intelligence, chapter 3.2, Structure-Mapping: A Theoretical Framework for Analogy, pages 303-310. Morgan Kaufmann Publishers, Inc., San Mateo, CA, 1988.

[5] International Organization for Standardization, Geneva. ISO 8879:1986 Information processing - Text and office systems - Standard Generalized Markup Language (SGML), October 1986.

[6] Robert L. Kelsey, Roger T. Hartley, and Robert B. Webster. An object-based methodology for knowledge representation. Technical Report LAUR 97-1598, Los Alamos National Laboratory, Los Alamos, NM, 1997. submitted to ACM CIKM 1997.

[7] Eve Maler and Jeanne El Andaloussi. Developing SGML DTDs From Text to Model to Markup. Prentice Hall PTR, Upper Saddle River, NJ, 1996.

[8] Elaine Rich and Kevin Knight. Artificial Intelligence. McGraw-Hill, Inc., New York, NY, second edition, 1991.

[9] Stuart J. Russell and Peter Norvig. Artificial Intelligence A Modern Approach. Prentice Hall, Englewood Cliffs, NJ, 1995.

[10] Roger C. Schank and Robert P. Abelson. Scripts Plans Goals and Understanding An Inquiry Into Human Knowledge Structures. Lawrence Erlbaum Associates, Publishers, Hillsdale, NJ, 1977.

[11] Eric van Herwijnen. Practical SGML. Kluwer Academic Publishers, Boston, MA, second edition, 1994.

[12] Patrick Henry Winston. Artificial Intelligence. Addison-Wesley Publishing Company, Reading, MA, second edition, 1984. 


\section{A DTD For The Object-Based Methodology}

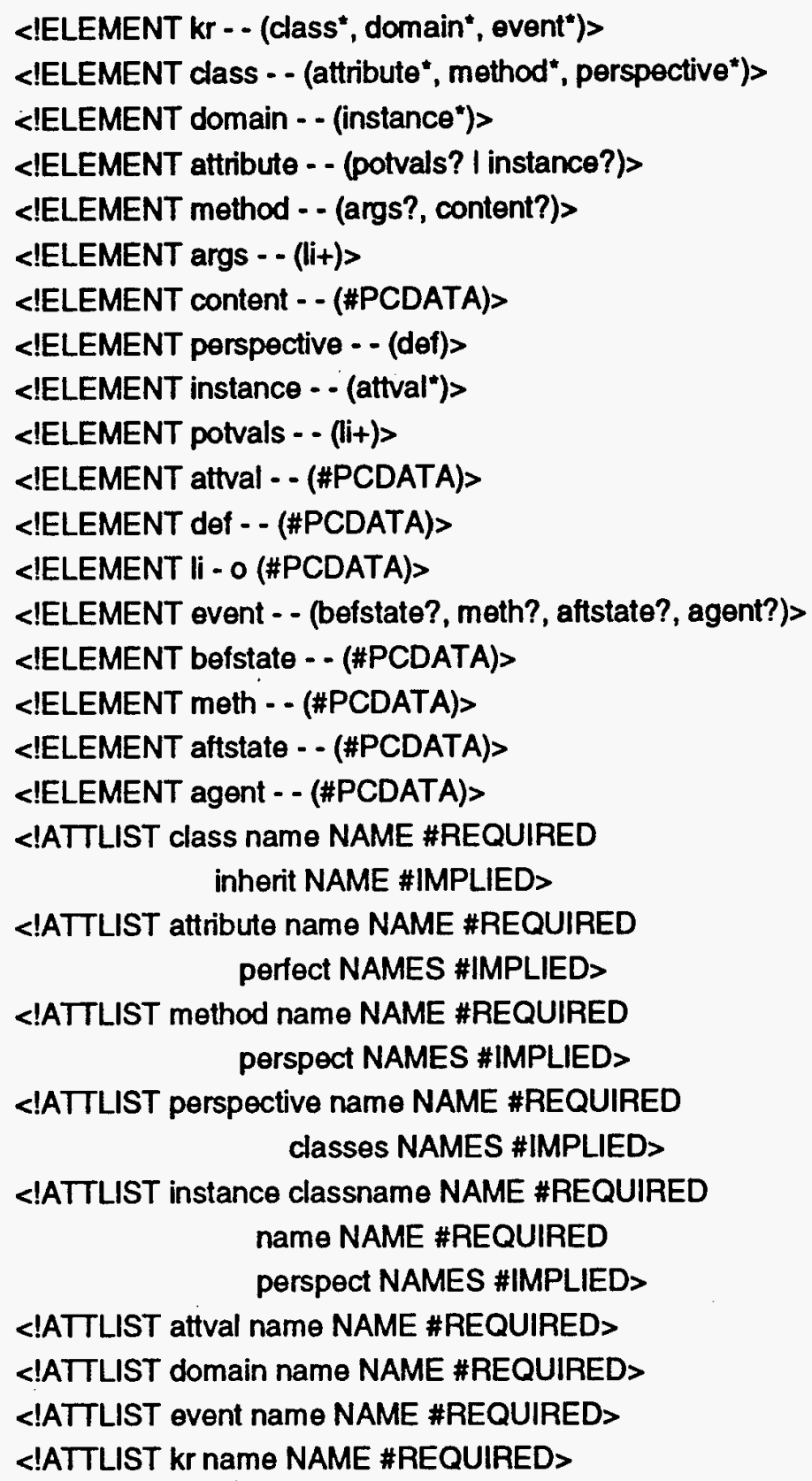


M97008299

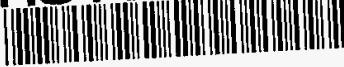

Report Number (14) $\frac{L A-U R--97-1828}{\operatorname{CONF}-971153--}$

Publ. Date (11) 199707

Sponsor Code (18) DOE/DP, XF

UC Category (19) UC-7OO, DOEIER

DOE 